\title{
BRAIN STATES MATTER. A REPLY TO THE UNFOLDING ARGUMENT.
}

\author{
JOHANNES KLEINER ${ }^{\dagger}$ \\ $\dagger$ Munich Center for Mathematical Philosophy \\ Ludwig-Maximilians-Universität München \\ Geschwister-Scholl-Platz 1, 80539 Germany
}

\begin{abstract}
Recently, it has been claimed that Integrated Information Theory and other theories of its type cannot explain consciousness ("unfolding argument"). We unravel this argument mathematically and prove that the premises of the argument imply a much stronger result according to which the observed problem holds for almost all theories of consciousness. We find, however, that one of the premises is unwarranted and show that if this premise is dropped, the argument ceases to work. Thus our results show that the claim of the unfolding argument cannot be considered valid. The premise in question is that measures of brain activity cannot be used in an empirical test of theories of consciousness.
\end{abstract}

Keywords: Theories of Consciousness; Models of Consciousness; Causal Structure; Integrated Information Theory, Recurrent Processing Theory

\section{INTRODUCTION}

In its modern form, the scientific study of consciousness aims to uncover the laws or regularities that link conscious experience with physical systems, such as the brain. Among the remarkable successes of this young field is the creation of various theories of consciousness, also referred to as models of consciousness (Seth, 2007). The most prominent examples are Integrated Information Theory (Oizumi et al., 2014; Marshall et al., 2016, Mayner et al., 2018), Recurrent Processing Theory (Lamme, 2006) and Global Neuronal Workspace Theory (Dehaene et al., 2011). These theories are being supplemented by a large body of philosophical theories about how the relation of conscious experience and the physical domain could be, e.g. the many variants of functionalism, representationalism or type identity theory.

The unfolding argument presented in Doerig et al. (2019) claims that some of the leading models of consciousness "are either false or outside the realm of science" (p. 56). The models in question are so-called causal structure theories, which define a system's experience in terms of the mutual interaction of its parts. If this claim is true, it has large consequences for the scientific study of consciousness, as Integrated Information Theory and Recurrent Processing Theory, two of the most promising contemporary models of consciousness, both qualify as causal structure theories.

In Sections 2.1, 2.2 and 2.3, we review the unfolding argument and state it in clear formal language. In Section 2.4, we prove that the premises used in the unfolding argument imply a much stronger result, namely that the problem identified in the unfolding argument holds for all models of consciousness that depend non-trivially 
on physical systems. This includes causal structure theories such as Integrated Information Theory, but also Global Neuronal Workspace Theory and any other model of consciousness which is functionalist or representationalist in nature or based on type identity theory. Finally, in Section 3 , we discuss one premise of the argument in detail and show that it is unwarranted both in a theoretical and practical sense. Readers who are not interested in the mathematical details of the unfolding argument can jump straight to Section 3 .

\section{The Unfolding Argument}

2.1. Setup. We start by explaining the setting in which the unfolding argument, and our generalization thereof, is staged. This setting indeed underlies many contemporary research programs in the scientific study of consciousness. A summary is given in Figure 1. Before introducing the underlying assumptions, we fix terminology and notation.

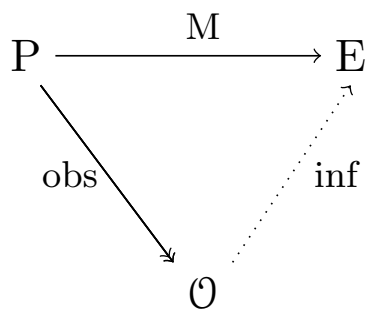

Figure 1. The setting of the unfolding argument. $P$ denotes a class of physical configurations, such as neural networks in a particular state, and $E$ denotes a set of states of experience. $\mathcal{O}$ denotes possible measurement results that can arise in experimental trials, i.e. observable datasets. The essential assumptions in Doerig et al. (2019) are that a) models of consciousness $M$ specify states of experience as a function of physical configurations and b) the results of measurements are determined by physical configurations alone. These assumptions determine arrows $M$ and obs in the diagram. Finally, the arrow inf corresponds to the process of inferring states of experience from observed datasets, e.g. from verbal reports. For further explanations and examples, see the main text.

In what follows, we denote by $P$ a class of configurations of physical systems. Any $p \in P$ denotes a model of the physical system, and we assume that this model contains all data necessary so that a particular model of consciousness can be applied. It should generally contain a particular choice of state for the system, e.g., and allow to determine the system's time evolution. We will simply refer to $p \in P$ as a configuration, and note that configurations of many different systems might be contained in $P$. To give an example, $P$ could comprise all configurations of neural networks, i.e. all different combinations of nodes, edges, states and update rules.

By $E$ we denote a space of states of experience, suitably chosen for a model of consciousness under consideration. If a model addresses the quantity or level of consciousness, e.g., $E$ could be chosen as the set of positive real numbers (including 0). If the model is made to describe whether a specific stimulus was perceived consciously or not, $E=\{0,1\}$. But in most cases, $E$ will have much more structure corresponding to 
the rich structure of experience. Various models of consciousness may refer to various aspects of experience, which is reflected in the varying mathematical structure of $E$.

Finally, we denote by $\hat{\mathcal{O}}$ a class of observables, i.e. quantities that can be measured in an experiment. Every observable comes with a set of possible measurement results. Measurement of several observables in an experimental trial yields a dataset that contains the results of the various measurements that have been performed. In what follows, we will work with the class of all such datasets, denoted by $\mathcal{O}$ and refer to them as observable datasets, because the datasets in this class can be observed in experimental trials.

The sets $\hat{\mathcal{O}}$ and $\mathcal{O}$ are two ways to describe the same choice, namely the choice of which observables to consider in an experimental trial or theoretical investigation. Different choices of observables $\hat{\mathcal{O}}$ determine different observable datasets $\mathcal{O}$, and from a given class $\mathcal{O}$ the underlying choice of observables $\hat{\mathcal{O}}$ can be abstracted. For reasons that will become clear below, we will exclusively work with the class $\mathcal{O}$ and refer to it as a choice of observable quantities. When explaining concepts, we will, however, often refer to the observables $\hat{\mathcal{O}}$ which underlie the choice of $\mathcal{O}$.

Operationally, a choice of observable quantities $\mathcal{O}$ corresponds to a choice of measurements that are to be considered, a specification of which measurements can be composed or concatenated, and the specification of all datasets that can result from such composition or concatenation. The important points are that every experimental trial yields one dataset $o \in \mathcal{O}$, and that every dataset $o \in \mathcal{O}$ can arise in some experimental trial.

An example for a choice of observable quantities relevant in what follows are inputoutput observables, i.e. all measurements that consist of the presentation of some input-stimulus and the subsequent recording of some output. Further details about the formalization of this concept will be given below.

We are now in a position to state the assumptions that underlie the setting of the unfolding argument. These assumptions are in fact wide-spread in parts of the scientific study of consciousness, which is why the unfolding argument is interesting. To have an unladen name, we will refer to them as the direct setting. We remark that throughout this paper, we will use the terms "model" and "theory" interchangeably.

Definition 2.1. The direct setting of the scientific study of consciousness consists of the following assumptions.

[A1] Models of consciousness specify states of experience as a function of physical configurations.

[A2] The results of observations are determined by physical configurations.

In order to analyse the unfolding argument, we need to work with the mathematical content of these assumptions. Mathematically, Assumption [A1] states that models of consciousness are functions $M: P \rightarrow E \mathbb{1}^{1}$ Assumption [A2] states that there is a

\footnotetext{
${ }^{1}$ We remark that some of the models of consciousness mentioned previously are not presented, to date, in formal/mathematical terms, so that the precise form of $f$ can only be estimated. Nevertheless, using the terminology of physical configurations and of states of experience, they amount to maps $f$ as described here. The form of $f$ does not have to be completely known for our results to apply, cf. Section 2.5
} 
correspondence $\AA^{2}$ obs : $P \rightarrow \mathcal{O}$, such that all possible datasets that can be observed in an experimental investigation of a system in configuration $p \in P$ are given by obs $(p)$. Put differently, the subset obs $(p) \subset \mathcal{O}$ comprises all datasets that can result in an experiment if the system $p$ pertains in the experiment.

The function $M$ and the correspondence obs give the corresponding arrows in Figure 1. In addition to the direct setting, the unfolding argument assumes that there is a function inf : $\mathcal{O} \rightarrow E$ which allows to infer states of experience from data that is being observed in an experiment. In contemporary experiments, the space of experience $E$ is often taken to be a set whose elements correspond directly to verbal reports, which is why this function inf is usually not mentioned or considered explicitly. But whether this function exists in certain paradigmatic cases is heavily debated (Lamme, 2006). The function inf is indicated by the dashed arrow in Figure 1 .

We remark that the distinction between states of experience and physical states in the direct setting is epistemological in nature, it does not necessarily require an ontological commitment to property dualism or similar metaphysical positions. This is so because there are "two fundamentally different methodological approaches that enable us to gather knowledge about consciousness: we can approach it from within and from without; from the first-person perspective and from the third-person perspective" (Metzinger, 1995). Correspondingly, there are two epistemically distinct notions of state, one associated with the third person perspective and one with the first person perspective. Even if a model is, say, physicalist in nature, so that states of experience are (ontologically) identical to certain physical properties, the model still needs to account for the two ways of gathering knowledge, represented by the two types of states.

2.2. The Problem. In order to explain the problem observed in Doerig et al. (2019), we consider the set of all those physical configurations that could have resulted in the dataset $o \in \mathcal{O}$. We denote this set as $P_{o}$. It is given by

$$
P_{o}:=\operatorname{obs}^{-1}(o) \text {, }
$$

where $\operatorname{obs}^{-1}(o)$ denotes the preimage of $o$, defined as

$$
\operatorname{obs}^{-1}(o):=\{p \in P \mid o \in \operatorname{obs}(p)\} .
$$

in the case of correspondences.

The observation underlying Doerig et al. (2019) is that if for a model of consciousness $M$ and a dataset $o \in \mathcal{O}$, it can be shown that there exists a $p \in P_{o}$ such that

$$
M(p) \neq \inf (o),
$$

there is a problem, because one of the following three cases applies: First, it could be the case that $\inf (o)$ points to the correct experience of the system $p \in P$. In this case, according to 2.3$)$, the model's prediction $M(p)$ is wrong, so that the whole model can be considered to be falsified. The same conclusion holds if neither $M(p)$ nor $\inf (o)$ point to the correct experience. The authors of Doerig et al. (2019), however, observe that even the third remaining case is problematic, for even if the model's prediction

\footnotetext{
${ }^{2} \mathrm{~A}$ correspondence is a generalization of a function. Whereas for a function $f: A \rightarrow B$, for every $a \in A$ there is exactly one element $f(a) \in B$, a correspondence $g: A \rightarrow B$ can specify more than one element for every $a \in A$ : The image $g(a)$ is a subset of $B$. Functions are special cases of correspondences, and both are best defined in terms of subsets of $A \times B$ (Encyclopedia of Mathematics, 2019).
} 
$M(p)$ were correct, 2.3$)$ implies that this prediction cannot be tested with respect to the dataset $o$.

This might not be considered reason enough to discard a model of consciousness if it is only the case for a small number of datasets, for situations where some predictions of a model are empirically inaccessible are common in various parts of science. However, if (2.3) holds for all datasets $o \in \mathcal{O}$, there certainly is a problem, because either there is at least one $o \in O$ for which $\inf (o)$ points to the correct experience, which implies as above that the model's prediction is wrong and hence the model should be considered falsified, or else, if $\inf (o)$ does not point to the correct experience for any $o \in \mathcal{O}$, no single prediction of the model can be tested, which means that the model is fully "outside the realm of science" (Doerig et al., 2019, p. 51). This is captured by the next definition.

Definition 2.2. A model of consciousness $M$ is empirically ill-defined with respect to a choice of observable quantities $\mathcal{O}$ if for every $o \in \mathcal{O}$, there is a $p \in P_{o}$ such that

$$
M(p) \neq \inf (o) .
$$

We remark that this definition refers to a choice of observable quantities $\mathcal{O}$. Only if a model $M$ is empirically ill-defined with respect to a sufficiently large set of observables, which captures all relevant experimental cases, can there be reason enough to discard this model as a scientific theory.

At the heart of the unfolding argument is the following lemma.

Lemma 2.3. If for every $o \in O$ there is a transformation $T: P_{o} \rightarrow P_{o}$ such that $M(T(p)) \neq M(p)$ for at least one $p \in P_{o}$, then $M$ is empirically ill-defined with respect to the choice of observable quantities $\mathcal{O}$.

Proof. For any $o \in \mathcal{O}$, let $p \in P_{o}$ denote the physical system assumed to exist in the lemma and define $p^{\prime}:=T(p)$. By definition, $p^{\prime} \in P_{o}$ as well. We thus have $M(p) \neq M\left(p^{\prime}\right)$, which implies that either $M(p)=\inf (o)$, so that $M\left(p^{\prime}\right) \neq \inf (o)$, or $M\left(p^{\prime}\right)=\inf (o)$, so that $M(p) \neq \inf (o)$, or none of the two. From this it follows that either $M\left(p^{\prime}\right) \neq \inf (o)$ or $M(p) \neq \inf (o)$, or both. Thus for any $o \in \mathcal{O}$, there is at least one $p \in P_{o}$ such that $(2.4)$ holds, i.e. Definition 2.2 applies.

In Doerig et al. (2019) and Hanson and Walker (2019), Lemma 2.3 is being applied with respect to a particular choice of observables $\mathcal{O}$, namely all stimulus-response measurements mentioned above, e.g., "stimuli in a visual masking experiment are inputs, and the outputs may be button presses, verbal reports or any other measure shown to reliably correlate with subjective reports" (Doerig et al., 2019, p. 51). Following Doerig et al. (2019), we will refer to these observables as input-output observables, where the input is the stimulus and the output is the report.

Definition 2.4. Assuming that the physical systems $P$ under consideration come with a well-defined notion of input and output, we denote by $\hat{\mathcal{O}}_{\text {io }}$ all observables that consist of the presentation of some input to a system and of the recording of the corresponding output. The class of all possible datasets that can result from such measurements is $\mathcal{O}_{\text {io }}$.

What is crucial about this choice of observables is that any measurement of the internal structure, internal state or internal dynamics of a physical system is being excluded. Any possible data in an experiment directed at testing a model of consciousness $M$ can, according to this choice, only result from presenting an input and 
recording the corresponding output. The justification for excluding internal measurements is explained in Argument (P4) in (Doerig et al., 2019, p. 53). We discuss it in Section 3 .

2.3. The Argument. In Doerig et al. (2019), the previous notions are applied to models of consciousness, such as Integrated Information Theory (IIT) (Oizumi et al., 2014) or Recurrent Processing Theory (RPT) (Lamme, 2006), which specify experience as a function of the "causal structure" of the physical system. Here, the term "causal structure" is used to refer to the mutual dependence of the time evolution of parts of the system, or in other words, to the way the various parts of a system interact.

The unfolding argument utilizes the fact that for any $o \in \mathcal{O}_{\text {io }}$, i.e. for any data about input-output relations of a physical system, there is a transformation $T: P_{o} \rightarrow P_{o}$ that maps a physical system with recurrent causal structure to a physical system with feedforward causal structure, and vice versa, without changing the input-output behaviour of this system (Oizumi et al. 2014). This transformation is called unfolding (Doerig et al., 2019), eponymous for the name of the unfolding argument. For a schematic depiction, cf. Figures 2 and A1 in Doerig et al. (2019). Since IIT is defined such that a physical system with feedforward causal structure does not have conscious experience, according to the theory, whereas systems with recurrent structure do, the premises of Lemma 2.3 are satisfied so that, according to the lemma, IIT is empirically ill-defined with respect to the input-output observables $\mathcal{O}_{i o}$. The same idea is applied to argue about RPT. The arguments given in Doerig et al. (2019) thus prove the following lemma:

Lemma 2.5. Any causal structure theory of consciousness is empirically ill-defined with respect to the choice $\mathcal{O}_{\text {io }}$ of observable quantities.

Lemma 2.5 is a special case of Lemma 2.9 which will be proven in the next section.

2.4. Generalization. We have noted before that the essential feature of the class of observables considered in Doerig et al. (2019) is that they do not take into account the internal structure of a system. Thus for every input-output dataset $o \in \mathcal{O}_{\text {io }}$, there is a large class $P_{o}$ of physical systems that yield this input-output structure. Among these is a system that consists of a long list of input-output relations (a "giant lookup-table" in philosophical terminology (Block, 1981)) and, upon presentation of some input, simply selects the corresponding output from this list. Since the physical systems in $P$ should in principle be physically realizable, we have to assume that the simple system under consideration has some type of mechanism responsible for the reading of the input and printing of the output. We denote this system by $p_{o}$.

Definition 2.6. Let $p_{o}$ denote the simplest physical system that, provided with the list $o \in \mathcal{O}_{\text {io }}$, reads an input and selects an output from the list.

There are two ways to interpret this definition. One the one hand, one could interpret it axiomatically, i.e. apply the definition with respect to any suitable given notion of 'simplest system'. The subsequent steps in the logical construction would then similarly refer to the given notion of 'simplest system' via this definition. While generally fine, the problem with this approach is that it is difficult to evaluate examples.

On the other hand, one could apply a particular choice of 'simplest system'. Using the fact, e.g., that the complexity (roughly, the non-simplicity) of a system can be quantified via the Kolmogorov complexity of its algorithmic description, one could 
define the 'simplest system' in a class to be that system whose algorithmic description has the smallest Kolmogorov complexity. ${ }^{3}$ However, similar to the above case, this leads to problems when evaluating examples, mostly because contemporary models of consciousness are, except for a few cases, not presented in a language that is formal enough in order to apply these concepts.

We avoid this dilemma by working with a combination of the two approaches, assuming that the simplest system considered in Definition 2.6 is a simple feedforward implementation of a tree sorting algorithm. Using Definition 2.6, we can now specify which models of consciousness are actually susceptible to an unfolding-type argument:

Definition 2.8. A model of consciousness $M$ depends non-trivially on physical systems if for every $o \in \mathcal{O}_{\text {io }}$, there is at least one physical system $p \in P_{o}$ that has a different state of experience, according to $M$, than $p_{o}$.

Thus a model of consciousness depends non-trivially on physical systems if the state of experience it determines for a $p \in P$ depends at least to some minimal degree on the internal structure, the internal dynamics or internal state of $p$. Put in simple terms, this is the case if the model actually "cares" about how a physical system looks beyond its input-output relation. The following lemma generalizes the Unfolding Lemma 2.5.

Lemma 2.9. Any model of consciousness that depends non-trivially on physical systems is empirically ill-defined with respect to the choice $\mathcal{O}_{\text {io }}$ of observable quantities.

We emphasize that this statement is only true in the setting introduced in Definition 2.1 and only holds with respect to the choice $\mathcal{O}_{\text {io }}$ of observable quantities or a subset thereof, cf. Sections 3 .

Proof. For any $o \in \mathcal{O}_{\text {io }}$, let $p \in P_{o}$ denote the physical system that has, according to the model $M$, a state of experience that is different from the state of experience of $p_{o}$. We thus have two systems $p, p_{o} \in P_{o}$ with $M(p) \neq M\left(p_{o}\right)$. As in the proof of Lemma 2.3, this either implies that $M(p) \neq \inf (o)$ or $M\left(p_{o}\right) \neq \inf (o)$ or both. Thus for any $o \in \mathcal{O}_{\text {io }}$, there is at least one $p \in P_{o}$ such that (2.4) holds. Therefore $M$ is empirically ill-defined with respect to the choice $\mathcal{O}_{\text {io }}$ of observable quantities.

2.5. Examples. Before we proceed to the discussion, we consider examples.

Example 2.10. Global Neuronal Workspace Theory. First, we consider Global Neuronal Workspace Theory (GNW) (Dehaene et al., 2011; Dehaene and Naccache, 2001; Dehaene et al., 1998). There is no formal version of the theory yet that would allow for mathematical assessment, but the papers that define the theory suggest that according to GNW, two necessary conditions need to be satisfied in order for a system to have conscious experience. First, the system needs to contain "two main computational spaces, each characterized by a distinct pattern of connectivity" (Dehaene

${ }^{3}$ This could amount, e.g., to the following definition.

Definition 2.7. Let $\tilde{P} \subset P$ denote those physical systems which, provided with an input-output list $o$ from the class $\mathcal{O}_{\text {io }}$, read the input and select the corresponding output from the list. We define the simplest system $\tilde{p} \in P$ to be a system whose algorithmic description is shortest among $P$ in a given model language.

For every $o \in \mathcal{O}_{\text {io }}$, the system $p_{o}$ defined in Definition|2.6 would then be equal to the system $\tilde{p}$ when equipped with the input-output list $o$. Note that the formulation of Definition $[2.7 \mid$ is such that $o$ does not count towards the Kolmogorov complexity of $\tilde{p}$. 
et al. 2011, p. 56). The first computational space is a "processing network, composed of a set of parallel, distributed and functionally specialized processors or modular subsystems subsumed by topologically distinct (...) domains with highly specific local or medium-range connections" (ibid.). The second computational space is a "a global neuronal workspace, consisting of a distributed set of (...) neurons characterized by their ability to receive from and send back to homologous neurons in other (...) areas (...) through long-range excitatory axons" (ibid.). The second condition is that "[t]he entire workspace is globally interconnected in such a way that only one such conscious representation can be active at any given time" (p. 58).

For $o \in \mathcal{O}_{\text {io }}$, consider a system $p \in P_{o}$ that satisfies these two conditions and has the input-output relation $o$. This is always possible since one may, by hand, add computational spaces in the right way to any given system. According to GNW, the system $p$ has conscious experiences.

The simplest physical system $p_{o}$ defined in Definition 2.8, however, does not posses either of the computational spaces and in particular no global workspace. Thus it does not satisfy either of the two necessary conditions for consciousness, and has, according to GNW, no conscious experience at all. From this it follows that for every $o \in \mathcal{O}_{\text {io }}$ there is at least one physical system $p$ that has a different state of experience, according to GNW, than $p_{o}$, so that GNW theory depends non-trivially on physical systems. Thus by Lemma 2.9. GNW is empirically ill-defined with respect to $\mathcal{O}_{\text {io }}$.

We note that this is a first example of where Lemma 2.9 is stronger than Lemma 2.5 . because depending on how one reads the postulates of GNW, one may hold that a feedforward system may still have a global workspace (Doerig et al., 2019, Sec. 2.6), so that the unfolding transformation considered in Doerig et al. (2019) may not necessarily change the state of experience. However, the stronger Lemma 2.9 shows that GNW theory is nevertheless empirically ill-defined with respect to $\mathcal{O}_{\mathrm{io}}$, provided the assumptions of the unfolding argument hold.

Example 2.11. Integrated Information Theory. Our next example is Integrated Information Theory. Since the system $p_{o}$ is by assumption feedforward, and since IIT is built in such a way that a feedforward system has no conscious experience whatsoever, whereas a system that displays recurrent behaviour is conscious at least to a minimal degree, it is enough to show that for any $o \in \mathcal{O}_{\text {io }}$, there is a physical system that yields input-output data $o$ and is recurrent. This is possible by simply adding a recurrent part to the system $p_{o}$ (for examples cf. e.g. (Doerig et al., 2019, Appendix A and B)). Thus IIT depends non-trivially on physical systems and hence, by Lemma 2.9, it is empirically ill-defined with respect to the choice $\mathcal{O}_{\text {io }}$ of observables quantities if the assumptions of the direct setting hold true.

Example 2.12. Behaviourist Theories. A class of theories of consciousness that depend trivially on physical systems are behaviourist theories (Hauser, 2019). These theories are built on the assumption that conscious experience (among other things) can be explained completely based on stimulus-response data. Thus, by assumption, for any $o \in \mathcal{O}_{\text {io }}$, the state of consciousness of all configurations $p$ in the class $P_{o}$ is the

\footnotetext{
${ }^{4}$ One could argue that GNW theory should be formalized in a different manner which ensures that the even the simplest physical systems, such as lookup tables, exhibit global workspaces. If this were the case, the above argumentation would not apply. The question of what such a formulation would look like and whether it would be compatible with the cited passages of Dehaene et al. (2011); Dehaene and Naccache (2001); Dehaene et al. (1998) goes beyond the scope of this article.
} 
same. Since Definition 2.8 requires there to be at least one configuration in $P_{o}$ whose state of experience is different from all others, the condition required in the definition cannot hold. Hence, Lemma 2.9 does not apply.

Example 2.13. Functionalist Theories. Functionalism denotes a family of theories about the nature of mental states. Its core idea is that "a type of mental state is a type of causal role in a larger network linking inputs, outputs, and other internal states" (Kobes, 2014). A functionalist model of consciousness is, correspondingly, a concrete proposal of which functional role corresponds to which mental state.

What is essential here is that in addition to the input-output behaviour of a system, functionalist models relate to the (abstract) architecture of a system: A mental state is characterized by its relation to input stimuli, output behaviour and other mental states. The difference to causal structure theories as defined in Doerig et al. (2019) is that the latter consider the network of interactions of different parts of the physical system, whereas functionalism refers to the network of relations between different states of the system, input states and output states. To distinguish these two notions, we will use the term functional structure for the latter.

The defining criterion of functionalism is quite broad, and many different connotations of it exist. In order to evaluate Definition 2.8 mathematically, we will refer to one particular type of functionalism, which is sometimes called machine state functionalism (Levin, 2018). It identifies conscious experiences with the states of the finite automata that describes a given physical system.

A formal argument can be given quickly by utilizing the results of Hanson and Walker (2019). Note, however, that a formal argument may not be necessary. Functionalism developed as a response to problems of behaviourism (Levin, 2018), and an essential feature of overcoming these problems was to take into account some of the internal workings of a system. It is part of the conceptual definition of functionalist theories of consciousness that they depend non-trivially on physical systems.

The following argument shows formally that machine state functionalism depends non-trivially on physical systems. Let $o \in \mathcal{O}_{\text {io }}$ be some dataset that describes inputoutput relations, and let $p_{o}$ be the simplest physical system considered in Definition 2.6. Machine state functionalist models of consciousness depend non-trivially on physical systems if there is a physical system $p \in P_{o}$ with substantially different functional structure than $p_{o}$.

The task of proving that there is such as system, has recently been performed in Hanson and Walker (2019), where it has been shown that for a given finite automaton, there is a transformation, similar to the unfolding transformation, that changes the functional structure of that automaton from recurrent to feedforward and vice versa 5 Applying this transformation to the system $p_{o}$ yields a system $p \in P_{o}$ with the desired property, a substantially different functional structure. Machine state functionalism implies, then, that $p$ and $p_{o}$ have different states of experience, so that we may conclude that machine state functionalism depends non-trivially on physical systems, and hence, by Lemma 2.9, is empirically ill-defined with respect to the choice $\mathcal{O}_{\text {io }}$ of observable quantities.

\footnotetext{
${ }^{5}$ We remark that Hanson and Walker (2019) are discussing the functional structure (defined above) of a physical system, which is different from the causal structure. Thus, the results in this paper are, while very interesting, not directly applicable to IIT.
} 
Models of consciousness such as Global Workspace Theory (GWT) or Global Broadcasting Theory (Bayne et al., 2009) are functionalist in nature (Kobes, 2014). They all depend non-trivially on physical systems and hence are, according to Lemma 2.9. empirically ill-defined with respect to the choice $\mathcal{O}_{\text {io }}$ of observable quantities. This shows that a very large part of contemporary models of consciousness suffer from the same problem certified for IIT in Doerig et al. (2019).

Example 2.14. Causal Structure Theories. A theory is a causal structure theory, according to the terminology used in Doerig et al. (2019), if it makes essential use of "how parts of a system interact. If a system has the 'right' kind of causal structure, in other words, if its elements interact in the 'right' way, it is conscious. Otherwise, it is not" (Doerig et al., 2019, p. 52). Since the causal structure is, by definition, part of the internal specifications of a system, the state of experience specified by a causal structure theory for the system $p_{o}$ is different from the state of experience specified for some of the other systems $p \in P_{o}$ which shares the same input-output relations. This implies that causal structure theories depend non-trivially on physical systems, so that Lemma 2.9 is a generalization of the Unfolding Lemma 2.5.

\section{Discussion}

In the previous section we have mathematically shown that the problem identified in Doerig et al. (2019) does not only affect causal structure theories, but indeed all models of consciousness that depend non-trivially on physical systems. This includes Integrated Information Theory and Recurrent Processing Theory, but also Global Neuronal Workspace Theory or functionalist models of consciousness, to name just a few. Our results show that if the assumptions of the unfolding argument are valid, each and every one of those theories necessarily is either false or untestable.

Since this problem is so devastating, we need to consider the assumptions of the unfolding argument and our generalization in detail. Summarizing the last section, the assumptions are the following:

1. The argument makes two general assumptions, one about the type of model under consideration and one about how the results of experiments are determined in nature. These assumptions are explained in detail in Definition 2.1. They are in wide-spread use in theoretical neuroscience.

2. The argument assumes that states of consciousness of a system can be inferred from verbal report or similar outputs (such as button presses or behavioural indicators). This, too, is standard practice in the scientific study of consciousness.

3. Finally, the unfolding argument needs to assume that "measurement of brain activity itself or of other internal workings of the system" (Doerig et al., 2019 , p. 53) are excluded from empirical tests of models of consciousness.

That the last assumption is necessary for the unfolding argument might not be obvious. We explain in detail why this is so in Section 3.1. In Section 3.2, we examine the original justification of this assumption in Doerig et al. (2019). Finally, in Section 3.3 . we explain why this assumption is, in our view, unjustified.

Before considering this assumption in detail, we emphasize that there are only two options. Either all assumptions of the unfolding argument are valid, in which case our generalization shows that almost all models of consciousness would have to be discarded. Or at least one assumption is invalid, so that the unfolding argument is 
wrong in the sense that it fails to achieve what it claims to achieve. Both cases have substantial consequences, the first for the scientific study of consciousness as a whole, the second for the further discussion of the unfolding argument. In our opinion, the latter option pertains.

3.1. Why the unfolding argument breaks down. In this section, we show why the unfolding argument as presented in Doerig et al. (2019) breaks down if internal measurements are not strictly excluded from empirical tests of models of consciousness. We first explain this in terms of the original terminology of the paper, and subsequently in terms of the more formal terminology introduced in Section 2 .

Consider the following summary of the unfolding argument, given by the authors themselves (Doerig et al., 2019, p. 53):

(P1) In science we rely on physical measurements (based on subjective reports about consciousness).

(P2) For any recurrent system with a given input-output function, there exist feedforward systems with the same input-output function (and vice-versa).

(P3) Two systems that have identical input-output functions cannot be distinguished by any experiment that relies on a physical measurement (other than a measurement of brain activity itself or of other internal workings of the system).

(P4) We cannot use measures of brain activity as a-priori indicators of consciousness, because the brain basis of consciousness is what we are trying to understand in the first place.

(C) Therefore, EITHER causal structure theories are falsified (if they accept that unfolded, feedforward networks can be conscious), OR causal structure theories are outside the realm of scientific inquiry (if they maintain that unfolded feedforward networks are not conscious despite being empirically indistinguishable from functionally equivalent recurrent networks).

The assumption that internal measurements are strictly excluded enters in assumption (P3), in the restriction to "any experiment that relies on a physical measurement (other than a measurement of brain activity itself or of other internal workings of the system)" (emphasis added). Dropping the term in brackets renders statement (P3) wrong, because if experiments are allowed to measure brain activity or other internal workings of the system, two systems that have identical input-output functions (P2) can be distinguished by an experiment that relies on a physical measurement (contrary to what (P3) says). Put in terms of (C), unfolded feedforward networks are not empirically indistinguishable from functionally equivalent recurrent networks if internal measurements are allowed. Thus the simple either-or distinction of $(\mathrm{C})$ breaks down. Statement (P4) is a justification of the restriction considered in (P3) which we will discuss in the next section.

Mathematically this is reflected in the fact that Lemma 2.5 and Lemma 2.9 establish empirical ill-definedness only for the choice $\mathcal{O}_{\text {io }}$ of observables quantities, where $\mathcal{O}_{\text {io }}$ is defined precisely by the exclusion of internal measurements of any sort. If one considers a set that is larger than $\mathcal{O}_{\text {io }}$, e.g. by including internal measurements, the lemmas simply don't apply.

The last two paragraphs show that the presentation of the unfolding argument in Doerig et al. (2019), as well as its formal reconstruction in Section 2, presume the exclusion of internal measurements. One might proceed to ask two further questions: 
What goes wrong if internal measurements are not excluded? And can the argument be mended to work without this exclusion?

There are situations where the answer to the first question is straightforward. If internal measurements are allowed, unfolded feedforward networks can empirically be distinguished from functionally equivalent recurrent networks. Thus the simple eitheror distinction of the conclusion (C) mentioned above breaks down.

This can be illustrated in detail in the formal reconstruction of the argument presented in the previous section: If one replaces $\mathcal{O}_{\text {io }}$ by a set $\mathcal{O}$ of observable quantities that includes input-output measurements as well as internal measurements, the set $P_{o}$ defined in (2.1) becomes much smaller. If one allows for all possible internal measurements, it contains one physical configuration alone. Whereas for $o \in \mathcal{O}_{\text {io }}$, the requirement for a physical system $p$ to be in $P_{o}$ was only that $p$ has the input-output relation $o$, so that many physical systems will be contained in $P_{o}$, in the case of $o^{\prime} \in \mathcal{O}$, the requirement is much stronger. A physical system $p$ is in $P_{O^{\prime}}$ only if its inputoutput relation and internal measurements are as in $o^{\prime}$. This, in turn, implies that unfolding transformations $T$ as in Lemma 2.3 will cease to exist. The sets $P_{o^{\prime}}$ are simply too small to allow for transformations that preserve the observed data but satisfy $M(T(p)) \neq M(p)$. In physical terms, we may say that internal measurements break the invariance under unfolding transformations. If internal measurements are included, the system ceases to be a black box.

The answer to the second question is not straightforward, as a large number of additional assumptions and alternative strategies of argumentation might be considered, each of which would require a careful justification. The onus of proposing an alternative argument that is not prone to our criticism, however, lies with the proponents of the argument.

3.2. The original justification. The previous section has established that if internal measurements cannot be excluded from empirical tests of models of consciousness, the unfolding argument is wrong, in the sense that it does not establish what it claims to establish. This brings us to study the original justification in Doerig et al. (2019) of why internal measurements should be excluded from experimental tests. This justification is given in (P4) above: "( $\mathrm{P} 4)$ We cannot use measures of brain activity as a-priori indicators of consciousness, because the brain basis of consciousness is what we are trying to understand in the first place" (Doerig et al., 2019, p. 53).

We need to consider this statement carefully. The second part of the statement, "the brain basis of consciousness is what we are trying to understand ", is certainly true. Clearly, when considering models of consciousness such as IIT or GNW or general theories such as functionalism or type identity theory, we're trying to understand how physical structure ("the brain basis") relates to consciousness. The claim is that this implies that we cannot use "measures of brain activity as a-priori indicators of consciousness". But neither a justification for why this implication should hold, nor a specification of what "a-priori indicators of consciousness" are taken to be here is given.

In order to understand the intuition behind this statement, we thus need to turn to an example given in order to support (P4). Consider two robot systems with the same input-output relation, one of which has feedforward causal structure, whereas the other has recurrent causal structure. Then, according to Doerig et al. (2019), "proponents of IIT may still insist that the robot with $\phi=0$ is unconscious whereas 
the one with $\phi>0$ is conscious, owing to their differing causal structure. However, such a proposition quickly ends up in circularity because we have no criteria to settle the matter." What one may not do, according to this example, is to infer the state of consciousness of the robots by using internal measurements together with IIT. This makes sense, for this inferred state of experience and IIT's prediction of the state of experience would always align, so that the theory cannot be falsified. Inference and prediction would amount to the same procedure, which indeed is circular.

Put in more abstract terms, what one may not do in an empirical test of a model of consciousness, according to this example, is to infer the state of consciousness of some system from internal measurements using the very model that is to be tested. This makes sense and specifies both the meaning of "a-priori indicators of consciousness" and why (P4) holds. Importantly, however, it does not imply that one cannot use internal measurements in predictions of the state of consciousness of the system. As long as there are independent criteria that allow to infer the state of experience of the system, there is no circularity. Thus ( $\mathrm{P} 4)$ cannot be taken as a justification of the bracket in (P3). Internal measurements cannot be systematically excluded from empirical tests of models of consciousness.

Before moving on, we'd like to point out that the general idea of the authors of Doerig et al. (2019) considered in this section is important. Empirical tests of models of consciousness such as IIT or GNW on non-human subjects face methodological problems that tests on human subjects do not face, most notably the question of how one should infer the actual state of consciousness of these systems. These methodological problems may well be detrimental. But they do not imply that measurements of brain activity or of other internal structure can be systematically excluded. At the very least, measurements of brain states of human subjects need to be included.

3.3. Brain states matter. In the previous section, we have seen that Doerig et al. (2019) does not provide a valid justification of why internal measurements cannot be used in experimental tests of a model of consciousness.

In order to finish our argument, we now need to show why internal measurements may in fact be used in empirical tests of models of consciousness. We do this by describing a simple methodological scheme that uses internal measurements. This scheme is not new but in fact used in many empirical investigations to date, e.g. Kim et al. (2018); Sarasso et al. (2015). The validity of this scheme is founded in the validity of a falsificationist stance in the scientific study of consciousness, according to which proposed models of consciousness should be tested empirically by checking whether their predictions hold true ${ }^{6}$ Different models may of course make different predictions, and in what follows we consider only one particular type of prediction made by the models considered in the unfolding argument.

Falsificationism contrasts with an inductive approach to the scientific study of consciousness, cf. Remark 3.1, and it is conceivable that some would reject the use of falsifications in the scientific study of consciousness completely. The consideration of falsifications can, however, be justified directly with the unfolding argument, which is itself built on falsifications (cf. e.g. conclusion (C) quoted above). Thus if one were to reject falsificationism completely in the context of models of consciousness, one would have to reject the unfolding argument as well.

\footnotetext{
${ }^{6}$ Further exploration of the role of falsification in the scientific study of consciousness is carried out in Kleiner and Hoel 2020).
} 
Models of consciousness are formal hypotheses about how consciousness relates to the physical domain, e.g. to brain structure. The models of consciousness considered in the unfolding argument determine the conscious experience of a system in terms of its physical configuration (cf. Assumption [A1]]). Hence the conscious experience of a particular physical configuration is a prediction of a model of consciousness under consideration.

This prediction can be tested in cases where there are independent principles that allow to infer the conscious experience of these physical systems. These independent principles are typically given by interpretations of verbal reports, button presses or similar indicators. They may depend on the system under consideration, but this is not of importance for this section. What matters is that one can compare the above mentioned prediction of the state of consciousness of some model with the state of consciousness one has inferred from reports. If these two agree, the prediction is okay. If they disagree, one has found a falsification of the model under consideration, so that the model in its current form would need to be discarded.7

Crucially, for any model of consciousness that depends non-trivially on physical systems, the prediction needs to be based on data about the internal structure, internal state or internal dynamics of the system. This is so for IIT, GNW, functionalist theories or other examples discussed in Section 2.4. There may be other means to evaluate these models, but to make a prediction, an experimenter needs to carry out internal measurements.

This methodological procedure is the paradigm of testing IIT's prediction of the level of consciousness $\Phi(p)$ of a physical configuration $p$. But it is also considered, e.g., in contexts of GNW: "predicted 'signatures' of conscious access include a sudden, late and sustained firing in GNW neurons [referred to as conscious 'ignition'], a late sensory amplification in relevant processor neurons, and an increase in high-frequency oscillations and long-distance phase synchrony" (Dehaene et al., 2011, p. 58). Thus, as in IIT, one can falsify GNW by measuring brain activity in human subjects while presenting stimuli. If the subject were to report conscious access but the internal measurements would not show conscious ignition, the theory would be falsified. This is the exact same procedure as used for IIT, with the only difference that in the case of IIT, complex calculations are necessary to make the prediction of $\Phi(p)$, whereas for GNW, at present, the prediction can directly be stated: If there is no conscious ignition, there is no conscious access.

Thus internal measurements may, and in the case considered here, need to be used to empirically test models of consciousness. This is the origin of the slogan "brain states matter". A falsification of a model of consciousness considered in the unfolding argument may use internal measurements.

3.4. Upshot. In summary, we have shown the following:

1. If the strict exclusion of internal measurements in experimental tests is being dropped, the unfolding argument as stated in Doerig et al. (2019) breaks down. (Section 3.1)

2. The justification in Doerig et al. (2019) of why internal measurements need to be strictly excluded from experimental tests is not valid. (Section 3.2)

\footnotetext{
${ }^{7}$ This is an idealized description. In scientific practice falsification playes a somewhat less crucial role than often assumed, cf. e.g. Lakatos (1978).
} 
3. There is at least one valid experimental scheme that uses internal measurements. Thus internal measurements may not strictly be excluded when describing experimental tests. (Section 3.3)

From this it follows that the unfolding argument breaks down. This remedies all problems allegedly caused by the unfolding argument with respect to models of consciousness such as IIT. Put in a very concise form, we have shown that the conclusion drawn from the unfolding argument is based on an illegitimate restriction of the set of observables available to test a theory.

3.5. First-hand experience. We close this section by noting that in addition to internal measurements of a system, a yet different kind of observable could be considered when falsifying a theory of consciousness: first-hand experience. According to this idea, in addition to reports or behavioural indicators, a scientist would rely on his or her own conscious experience when testing a theory of consciousness.

There seem to be at least two variants of this idea. According to the isomorphism view detailed in Tsuchiya et al. (2019), the scientist would analyse his/her own conscious experience and use this analysis to try to justify or test a theory of consciousness, requiring that there is an isomorphism between the phenomenology of conscious experience, on the one hand, and the physical substrate, on the other. The existence of this isomorphism is seen as the major criterion for the validity of a theory of consciousness in this methodological program.

The other variant is that the scientist would go about constructing models of consciousness as usual (using reports and other behavioural indicators), but would try to falsify the theory also with respect to his/her own conscious experience. To falsify Recurrent Processing Theory, e.g., the scientist would have a TMS pulse applied to some area of his/her own brain to see whether the conscious perception of a stimulus breaks down as predicted by that theory.

As with internal measurements, the possibility of using first-hand experience in an experimental test of a model of consciousness entails that the relevant set of observable quantities is not $\mathcal{O}_{\text {io }}$, but in fact larger. Since neither the unfolding argument, nor its generalization, make any statement about this larger class, neither can be taken to imply that a model of consciousness is empirically ill-defined per se and should be discarded. We refer the reader to Tsuchiya et al. (2019) for a further discussion of first-hand experiences in the context of the unfolding argument.

Remark 3.1. The scientific study of consciousness is a fascinating field, not least because it is at the intersection of a huge number of disciplines spanning from neuroscience and cognitive psychology via physics and chemistry to philosophy and mathematics. These disciplines contribute valuable insights and results to the study of consciousness, but they also come with different empirical or theoretical methodologies and, most importantly, different conceptions of what the fundamental scientific method is or should be.

Consider, e.g., physics and neuroscience. Physics is almost exclusively working with the hypothetico-deductive approach, which is focused on falsifying predictions made on the basis of a hypothesis, i.e. on the basis of a formal theory about some phenomenon or on the basis of some mathematized ideas about how a phenomenon could behave. Where the hypothesis came from and whether it has a priori empirical support or not is, according to this methodology, not essential. It may have come from wild speculation, 
may have been a straightforward consequence of some other formal theory, or often is simply a formal expression of conceptual or philosophical ideas. What is essential, however, is that the hypothesis could be true, i.e. that it does not violate other elements of the canon of accepted knowledge unintentionally. Correspondingly, an essential part of the hypothetico-deductive approach is the working hypothesis that the theory under consideration is fundamentally true. This needs to be distinguished from and does not entail the claim that the theory is fundamentally true. Neuroscience, on the other hand, works in a much more inductive way, where theories or models are extracted, in a process of induction, from experimental data.

In the scientific study of consciousness, these two conceptions of the scientific methodology meet and, to some extent, clash. Whereas some researchers insist on an inductive procedure, which aims to infer the relation between conscious experience and physical states from experimental data, others work with a hypothetico-deductive approach and are willing to consider models of consciousness that are not induced in a strict sense from experimental observations.

Integrated Information Theory, e.g., falls into the hypothetico-deductive realm, its research program is to derive a theory of consciousness from introspection and general guiding ideas (Oizumi et al., 2014). Global Neuronal Workspace Theory, on the other hand, falls more into the inductive realm, since the theory is intimately tied to experimental observations and, to date, is formulated in terms of brain architecture (Dehaene et al., 2011).

A good example of the conflict that can arise if these ideas clash is Lau and Michel (2019), where IIT is criticized for trying to be a fundamental theory of consciousness on the basis of principles that feature highly in an inductive approach. Whether the various criticisms that are mentioned in Lau and Michel (2019) are adequate or not, it is important to note that the paper employs criteria from one approach to a theory that was built on criteria of the other. What looks like an "unscientific leap of faith" (Lau and Michel, 2019, p. 8) from one perspective may be an essential ingredient in making progress in another.

This all matters here because the criteria we use to asses whether a model of consciousness is promising or not depend strongly on which methodological background we have. This is true even more when it comes to judgements of whether some procedure or observable is empirically reasonable or not. The paradigm case for testing IIT's prediction explained in Section 3.3 e.g., is more aligned with the hypothetico-deductive approach than it is with the inductive approach. Correspondingly, researchers that prefer the latter might feel less willing to accept this procedure as reasonable.

The same is true for other concepts used in the scientific study of consciousness. Researchers with an inductive background, e.g., seem to think of a model of consciousness more in terms of a specification of which neural state is conscious, whereas researchers with a hypothetico-deductive background tend to think of models of consciousness more in terms of laws or bridging principles 8

What is important at present is that all approaches have merits and may prove to be useful in uncovering conscious experience. The scientific study of consciousness is a

\footnotetext{
${ }^{8}$ Mathematically, both amount to the consideration of maps of the form $f: P \rightarrow E$ or more general relations between $P$ and $E$. The specification of which neural state is conscious is essentially a map $f$ which specifies the predicate "conscious" or "unconscious" for each state. In the case of laws or bridging principles, the map or relation $f$ is the law or bridging principle, when expressed in mathematical terms.
} 
very young field, and which scientific method is most appropriate will have to be seen. Correspondingly, tests of a theory that are somewhat hypothetico-deductive in nature cannot simply be dismissed.

\section{Summary and Conclusion}

In this contribution, we have subjected the unfolding argument to a thorough formal analysis. Based on this analysis, we have shown that the premises of the unfolding argument entail a much stronger result than the one obtained in Doerig et al. (2019). If the premises are valid, the problem identified in the unfolding argument affects not only causal structure theories, but in fact all models of consciousness that depend non-trivially on physical systems. This includes, e.g., Integrated Information Theory, Global Neuronal Workspace Theory and any other model of consciousness that is functionalist in nature. Only models of consciousness that are compatible with the outdated stance of behaviourism can evidently escape our generalization.

Our results suggest that the unfolding argument and our generalization thereof are in fact no-go theorems, which exclude a set of assumptions rather than specific models, and lead us to analysing the assumptions in detail.

The most critical premise of the unfolding argument is the strict exclusion of internal measurements from experimental tests of models of consciousness. Any measurement that yields data about brain states or brain activity, such as EEG or fMRI, needs to be strictly excluded for the argument to work. We have shown in detail where this premise is being made in the argument, why it cannot be dropped and why it is unwarranted. This implies that the unfolding argument does not achieve what it claims to achieve, namely to show that "causal structure theories are either false or outside the realm of science".

Acknowledgements: I would like to thank Robin Lorenz and Robert Prentner for discussions on this topic and Adrien Doerig for discussion of parts of this manuscript. This analysis of the unfolding argument was originally conceived of as part of a larger contribution.

Funding: This research did not receive any specific grant from funding agencies in the public, commercial, or not-for-profit sectors.

\section{REFERENCES}

Bayne, T., Cleeremans, A., and Wilken, P. (2009). The Oxford companion to consciousness. Oxford University Press.

Block, N. (1981). Psychologism and behaviorism. The Philosophical Review, 90(1):543.

Dehaene, S., Changeux, J.-P., and Naccache, L. (2011). The Global Neuronal Workspace Model of Conscious Access: From Neuronal Architectures to Clinical Applications, pages 55-84. Springer Berlin Heidelberg, Berlin, Heidelberg.

Dehaene, S., Kerszberg, M., and Changeux, J.-P. (1998). A neuronal model of a global workspace in effortful cognitive tasks. Proceedings of the National Academy of Sciences, 95(24):14529-14534.

Dehaene, S. and Naccache, L. (2001). Towards a cognitive neuroscience of consciousness: basic evidence and a workspace framework. Cognition, 79(1):1 - 37. The Cognitive Neuroscience of Consciousness. 
Doerig, A., Schurger, A., Hess, K., and Herzog, M. H. (2019). The unfolding argument: Why IIT and other causal structure theories cannot explain consciousness. Consciousness and Cognition, 72:49 - 59.

Encyclopedia of Mathematics (2019). Correspondence. Springer.

Hanson, J. R. and Walker, S. I. (2019). Integrated information theory and isomorphic feed-forward philosophical zombies. Preprint.

Hauser, L. (2019). Behaviorism. In Internet Encyclopedia of Philosophy.

Kim, H., Hudetz, A. G., Lee, J., Mashour, G. A., Lee, U., Avidan, M. S., Bel-Bahar, T., Blain-Moraes, S., Golmirzaie, G., Janke, E., et al. (2018). Estimating the integrated information measure phi from high-density electroencephalography during states of consciousness in humans. Frontiers in human neuroscience, 12:42.

Kleiner, J. and Hoel, E. (2020). Falsification and consciousness. arXiv preprint arXiv:2004.03541.

Kobes, B. W. (2014). Functionalist theories of consciousness. In Bayne, T., Cleeremans, A., and Wilken, P., editors, The Oxford companion to consciousness. Oxford University Press.

Lakatos, I. (1978). The methodology of scientific research programmes. Cambridge University Press.

Lamme, V. A. (2006). Towards a true neural stance on consciousness. Trends in cognitive sciences, 10(11):494-501.

Lau, H. and Michel, M. (2019). On the dangers of conflating strong and weak versions of a theory of consciousness. Preprint, 10.31234/osf.io/hjp3s.

Levin, J. (2018). Functionalism. In Zalta, E. N., editor, The Stanford Encyclopedia of Philosophy. Metaphysics Research Lab, Stanford University, fall 2018 edition.

Marshall, W., Gomez-Ramirez, J., and Tononi, G. (2016). Integrated information and state differentiation. Frontiers in Psychology, 7:926.

Mayner, W. G. P., Marshall, W., Albantakis, L., Findlay, G., Marchman, R., and Tononi, G. (2018). Pyphi: A toolbox for integrated information theory. PLOS Computational Biology, 14(7):1-21.

Metzinger, T. (1995). The problem of consciousness. In Metzinger, T., editor, Conscious experience, pages 3-37. Imprint Academic.

Oizumi, M., Albantakis, L., and Tononi, G. (2014). From the phenomenology to the mechanisms of consciousness: Integrated Information Theory 3.0. PLOS Computational Biology, 10(5):1-25.

Sarasso, S., Boly, M., Napolitani, M., Gosseries, O., Charland-Verville, V., Casarotto, S., Rosanova, M., Casali, A. G., Brichant, J.-F., Boveroux, P., et al. (2015). Consciousness and complexity during unresponsiveness induced by propofol, xenon, and ketamine. Current Biology, 25(23):3099-3105.

Seth, A. (2007). Models of consciousness. Scholarpedia, 2(1):1328.

Tsuchiya, N., Andrillon, T., and Haun, A. (2019). A reply to "the unfolding argument": Beyond functionalism/behaviorism and towards a truer science of causal structural theories of consciousness. Preprint, 10.31234/osf.io/a2ms9. 